\title{
MODELING THE EFFECT OF A NEARBY BOUNDARY ON THE HRTF
}

\author{
Nail A. Gumerov and Ramani Duraiswami \\ Perceptual Interfaces and Reality Laboratory \\ Institute for Advanced Computer Studies, University of Maryland, College Park, MD 20742 \\ \{gumerov,ramani\}@umiacs.umd.edu
}

\begin{abstract}
Understanding and simplified modeling of the Head Related Transfer Function (HRTF) holds the key to many applications in spatial audio. We develop an analytical solution to the problem of scattering of sound from a sphere in the vicinity of an infinite plane. Using this solution we study the influence of a nearby scattering rigid surface, on a spherical model for the HRTF.
\end{abstract}

\section{INTRODUCTION}

Humans have the remarkable ability to localize a sound source using the sound received at just two receivers (ears), even in the presence of noise and other sources. Future spatial audio applications that wish to exploit this ability, such as those in virtual and augmented reality, will require a great degree of fidelity in rendering the sound localization cues to be perceptually convincing [1]. A key block to developing such fidelity is the present lack of understanding of the cues that arise from the scattering of sound off the external ears, head, and bodies of the person, and by scattering off surfaces in the environment. All of the acoustic cues resulting from scattering off the person are captured by a frequency response function called the head-related transfer function (HRTF). For a particular source location, the HRTF is defined as the ratio of the complex sound pressure level (SPL) at the eardrum to the SPL at a specified location when the listener is absent [2]. Thus, a primary goal of current research is to determine how complicated models that achieve perceptual fidelity have to be [3], and develop simple models and an understanding of the human HRTF [4].

The HRTF depends on many factors including the shape of the head and pinnae, spatial orientation of the head relative to the source of sound, the room environment, proportions of the body, and others. Such a multiparametric dependence of the HRTF makes the study and modeling of the HRTF extremely complex. There is need for simple modeling and experiments to elucidate its structure.

To use the HRTF in applications the influence of nearby boundaries on it must be determined. In all applications a wall is present, at least in the form of a ground plane. Similarly, many experiments performed to measure the HRTF often have a nearby surface or boundary, and its influence on the results must be assessed.

Recently Duda \& Martens [2] performed mathematical modeling for sound scattering from a sphere, and the HRTF induced including its close-range behavior. They verified their results with experimental data. This simple treatment allowed for insight, and resulted in a simple model for approximating the HRTF for applications [4]. The purpose of this paper is to study the influence

Support of NSF award 0086075 is gratefully acknowledged. We would also like to thank Prof. R.O. Duda for making available his program for the spherical HRTF model. of a nearby rigid wall (or floor) using an analytical technique we develop, that permits one to clearly distinguish the influence of any introduced parameters. We present our model problem and its solution in $\$ 2$, assess its properties for a few interesting configurations in $\S 3$, and conclude in $\S 4$ with a few remarks based on these solutions.

\section{PROBLEM DEFINITION AND SOLUTION}

PROBLEM STATEMENT: The geometry of the problem is shown in Fig. 1. There is a monochromatic point source at $A$ and a sphere of radius $a$, with center at $O$. These are respectively located at distances $h$ and $H$ from a rigid wall at $z=H$ in a half-space $z<H$, with $d$ the distance between the source and the sphere center. Note that the case of a non-monochromatic source can be treated using Fourier analysis with our results. The com-

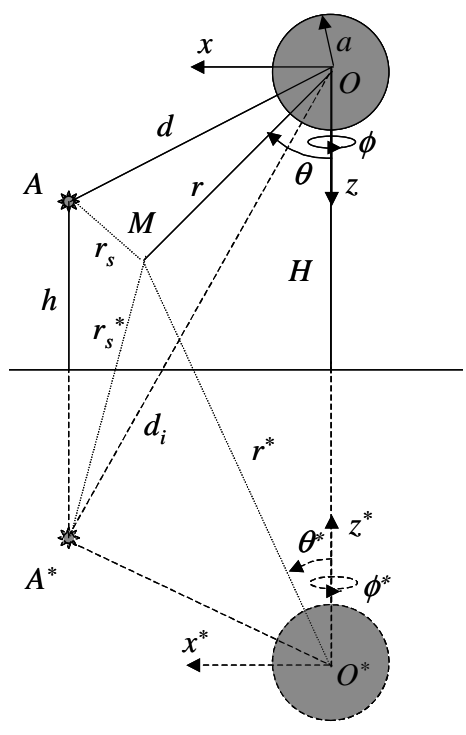

Fig. 1. Problem definition and notation.

plex pressure amplitude, $\Psi$, satisfies:

$$
\nabla^{2} \Psi+k^{2} \Psi=Q \delta\left(\mathbf{r}-\mathbf{r}_{r}\right)
$$

where $k=\omega / c$ is the wavenumber, $\omega$ the circular frequency, $c$ the sound speed, and $Q$ the source intensity. $\Psi$ is subject to the following boundary conditions on the rigid surfaces of the sphere, $S$, and the wall, $z=H$,

$$
\left.\frac{\partial \Psi}{\partial n}\right|_{S}=0,\left.\quad \frac{\partial \Psi}{\partial z}\right|_{z=H}=0 .
$$


Near the monopole source $\Psi$ satisfies

$$
\left.\Psi\right|_{M \rightarrow A} \sim Q G_{k}\left(r_{s}\right), \quad G_{k}\left(r_{s}\right)=\frac{e^{i k r_{s}}}{4 \pi r_{s}},
$$

where $r_{s}$ is the distance between the source and a field point $M$, $G_{k}$ the free space Green's function. Far from the source and the sphere the Sommerfeld radiation condition applies:

$$
\lim _{r \rightarrow \infty} r\left(\frac{\partial \Psi}{\partial r}-i k \Psi\right)=0 .
$$

Consider now two solutions to the problem at a field point $M$ : in the presence of the sphere, $\Psi(M)$, and absence of the sphere, $\Psi_{0}(M)$. We define the HRTF, $\mathbb{H}$, at any point $M$ on $S$ as the ratio:

$$
\mathbb{H}(M)=\frac{\Psi(M)}{\Psi_{0}(M)}, \quad M \in S .
$$

Note that this definition of the HRTF differs from that in [2]. That definition can be recovered by multiplying $\mathbb{H}$ by $\Psi_{0}(M) / \Psi_{0}(O)$.

SOLUTION: To solve the problem we replace the plane of symmetry by an image source and image sphere. We introduce two reference frames - at the sphere center, $O$, and at the center of the image sphere, $O^{*}$. The 2 nd frame is a mirror image of the 1 st frame, and all coordinates in it are marked with a $*$ superscript. The axis $z$ is directed along $O O^{*}$. The coordinates of the source and its image in the frame at $O$ are $\mathbf{r}_{r}=\left(d, \theta_{r}, \phi_{r}\right)$ and $\mathbf{r}_{i}=\left(d_{i}, \theta_{i}, \phi_{i}\right)$. The solution to (1) can be written as:

$$
\Psi=Q\left[G_{k}\left(\left|\mathbf{r}-\mathbf{r}_{r}\right|\right)+G_{k}\left(\left|\mathbf{r}-\mathbf{r}_{i}\right|\right)+\psi(\mathbf{r})+\psi\left(\mathbf{r}^{*}\right)\right] .
$$

$\psi$ is regular for $r \geq a$, and has the multipole expansion:

$$
\psi=\sum_{n=0}^{\infty} \sum_{m=-n}^{m=n} A_{n}^{m} S_{n}^{m}(\mathbf{r}), S_{n}^{m}(\mathbf{r})=h_{n}(k r) Y_{n}^{m}(\theta, \phi) \text {. (7) }
$$

Here $A_{n}^{m}$ are coefficients, $S_{n}^{m}(\mathbf{r})$ is a multipole of order $n$ and degree $m, h_{n}$ are spherical Hankel functions of the 1st kind that satisfy the Sommerfeld condition, and $Y_{n}^{m}(\theta, \phi)$ are orthonormal spherical harmonics. From now on, to conserve space, we will suppress the argument for the $Y_{n}^{m}$, except when necessary to indicate the reference frame for $\theta \& \phi$, e.g., we indicate $Y_{n}^{m}\left(\theta_{i}, \phi_{i}\right)$ as $Y_{n}^{m}[i]$. For $|\mathbf{r}| \leqslant d$ the 1 st term in Eq. (6) can be expanded as:

$$
G_{k}\left(\left|\mathbf{r}-\mathbf{r}_{r}\right|\right)=i k \sum_{n=0}^{\infty} j_{n}(k r) h_{n}(k d) \sum_{m=-n}^{m=n} \bar{Y}_{n}^{m}[r] Y_{n}^{m},
$$

where $j_{n}$ are spherical Bessel functions of the 1 st kind and $\bar{Y}_{n}^{m}$ are complex conjugate to $Y_{n}^{m}$. The same equation holds for $G_{k}\left(\left|\mathbf{r}-\mathbf{r}_{i}\right|\right)$ but with $d_{i}, \theta_{i}$, and $\phi_{i}$ instead. To expand the latter term in Eq. (6), we use the following representation valid for $|\mathbf{r}| \leqslant 2 H$ :

$$
S_{n}^{m}\left(\mathbf{r}^{*}\right)=h_{n}\left(k r^{*}\right) Y_{n}^{m}[*]=\sum_{l=|m|}^{\infty} \nu_{l n}^{m} j_{l}(k r) Y_{l}^{m}
$$

where the order of spherical harmonics, $m$, does not change due to the selection of the reference frames $\left(\phi^{*}=\phi\right) ; \nu_{n l}^{m}$ are the coefficients of the re-expansion depending on $k H$ alone. Their computation is key to the solution [7]. Using Eq. (7) and (8) we have

$$
\psi\left(\mathbf{r}^{*}\right)=\sum_{n=0}^{\infty} \sum_{m=-n}^{m=n} A_{n}^{m} \sum_{l=|m|}^{\infty} \nu_{l n}^{m} j_{l}(k r) Y_{l}^{m} .
$$

Using the fact that $Y_{l}^{m}=0$ for $|m|>l$ we can regroup the summation and represent $\Psi(\mathbf{r})$ as:

$$
\begin{aligned}
& \Psi(\mathbf{r})=Q \sum_{n=0}^{\infty} \sum_{m=-n}^{m=n}\left\{\mathbb{B}_{n}^{m} j_{n}(k r)+A_{n}^{m} h_{n}(k r)\right\} Y_{n}^{m} \\
& \mathbb{B}_{n}^{m}=i k\left(h_{n}(k d) \bar{Y}_{n}^{m}[r]+h_{n}\left(k d_{i}\right) \bar{Y}_{n}^{m}[i]\right)+\sum_{l=0}^{\infty} \nu_{n l}^{m} A_{l}^{m} .
\end{aligned}
$$

Satisfying (2) on the sphere and using the orthogonality/completeness of the $Y_{n}^{m}$ we get a linear system for the $A_{n}^{m}$. Writing the $A_{n}^{m}$ as a column vector $\mathbf{A}^{m}=\left\{A_{n}^{m}\right\}$ we can write this system as:

$$
\mathbf{M}^{m} \mathbf{A}^{m}=\mathbf{B}^{m}, \quad \mathbf{M}^{m}=\mathbf{I}+\mathbf{N}^{m}, \quad m=0, \pm 1, \ldots
$$

where $\mathbf{I}=\left\{\delta_{n l}\right\}$ is the identity, and $\mathbf{N}^{m}=\left\{N_{n l}^{m}\right\}$ and the components of the r.h.s. vector $\mathbf{B}^{m}=\left\{B_{n}^{m}\right\}$ are:

$$
\begin{aligned}
N_{n l}^{m} & =\frac{j_{n}^{\prime}(k a)}{h_{n}^{\prime}(k a)} \nu_{n l}^{m}, \\
B_{n}^{m} & =-i k \frac{j_{n}^{\prime}(k a)}{h_{n}^{\prime}(k a)}\left[h_{n}(k d) \bar{Y}_{n}^{m}[r]+h_{n}\left(k d_{i}\right) \bar{Y}_{n}^{m}[i]\right]
\end{aligned}
$$

Note that for the $m$ th degree system, $B_{n}^{m}=0, \nu_{n l}^{m}=0$ for $n<$ $|m|$ and $l<|m|$. Therefore $A_{n}^{m}=0$ for $n<|m|$ and equation (11) applies only for $n \geqslant|m|$. The system of equations can be solved by truncating the infinite matrices $\mathbf{N}^{m}$ and vectors $\mathbf{A}^{m}$ and $\mathbf{B}^{m}$ by their initial $L$ components. This results in an approximate solution. However the series converge rapidly, and the choice of $L$ is made by determining when additional terms make no difference.

An approximation can be made in the case the influence of the field scattered by the image sphere is small compared to the incident fields generated by the source and its image. In this case solution of Eq. (11) can be represented as:

$$
\mathbf{A}^{m}=\left(\mathbf{I}+\mathbf{N}^{m}\right)^{-1} \mathbf{B}^{m}=\left(\mathbf{I}-\mathbf{N}^{m}+\ldots\right) \mathbf{B}^{m} .
$$

The zeroth-order approximate solution is $\mathbf{A}^{m}=\mathbf{B}^{m}$. Knowing $\mathbf{A}^{m}, \Psi(\mathbf{r})$ can be determined on the sphere. Eq (10) at $r=a$ can be substantially simplified if the $\mathbb{B}_{n}^{m}$ terms in (10) are expressed via coefficients $A_{n}^{m}$ according to (11) using the Wronskian $W\left\{j_{n}(k a), h_{n}(k a)\right\}=j_{n}(k a) h_{n}^{\prime}(k a)-j_{n}^{\prime}(k a) h_{n}(k a)=$ $i(k a)^{-2}$. This results in:

$$
\left.\Psi\right|_{r=a}(\theta, \phi)=\frac{Q}{i k^{2} a^{2}} \sum_{n=0}^{\infty} \sum_{m=-n}^{m=n} A_{n}^{m} \frac{Y_{n}^{m}}{j_{n}^{\prime}(k a)}
$$

COMPUTATIONAL PROCEDURE: For rapid computation of the coefficients of the multipole reexpansion, $\nu_{n l}^{m}(2 k H)$, we derived several recurrence and symmetry relations, that are omitted for reasons of space, and will be presented elsewhere [7]. Computation of the matrix $\mathbf{N}^{m}$ as well as $\mathbb{H}$ requires computation of Hankel, Bessel and associated Legendre functions. This is performed using standard recurrence relations (e.g. see [5, 6]). Note that $\mathbf{M}^{m}$ depends on $k H \& k a$ only, and can be decomposed once for fixed $k H \& k a$. 


\section{NUMERICAL STUDY OF THE WALL INFLUENCE}

The pressure on the sphere surface $\Psi(\theta, \phi)$ depends on the ratio of the sphere size to the characteristic wavelength, $k a$, and the three length ratios, $d / a, h / a$, and $H / a$, characterizing the geometry. These form a 4 dimensional parameter space. We present results obtained by varying these parameters to illustrate the major effects.

The definition of the HRTF requires comparison of the scattered sound signal to a standard signal (in the absence of the head), $\Psi_{0}$ in (5). In the present problem, if we choose the same $\Psi_{0}$ as in [2] the HRTF exhibits features which, while explainable, can be hard to understand intuitively. Thus, to aid physical understanding we introduce several different transfer function definitions. In the form written in (5), where $\Psi_{0}$ is taken at the same point in the presence and in the absence of the head, $\mathbb{H}(M)$ shows the effect of the head on the acoustic field. We can decompose the influence of all factors on this composite HRTF. For example we can normalize the HRTF with the free space solution, $Q G_{k}(d)$, to show the influence of the head width and the wall as

$$
\begin{aligned}
\mathbb{H}^{00}(M) & =\frac{\Psi(M)}{Q G_{k}(d)}=\frac{\Psi(M)}{\Psi_{0}(M)} \frac{\Psi_{0}(M)}{\Psi_{0}(O)} \frac{\Psi_{0}(O)}{Q G_{k}(d)} \\
& =\mathbb{H}(M) \mathbb{H}_{O}^{M} \mathbb{H}_{00}^{O}=\mathbb{H}(M) \mathbb{H}_{00}^{M}
\end{aligned}
$$

Here $\mathbb{H}_{O}^{M}=\Psi_{0}(M) / \Psi_{0}(O)$ is the ratio of the pressure at the two points $M$ and $O$ in the absence of the head, $\mathbb{H}_{00}^{O}=$ $\Psi_{0}(O) / Q G_{k}(d)$ is the ratio of the pressure at the head center in the presence and absence of the wall (without the head). These two effects can be combined in one factor $\mathbb{H}_{00}^{M}=\mathbb{H}_{O}^{M} \mathbb{H}_{00}^{O}$, which can be interpreted as the effect of the scattering not related to the head. The amplitude of the HRTF are usually is measured in dBs. Thus when expressed logarithmically these contributions sum up.

The effect of the wall on the acoustic field can be decomposed into two effects. The first effect is the effect of the image source, and the second effect is that of scattering from the image sphere. Mathematically, if the second effect is negligible, we can replace the matrix $\mathbf{M}^{m}$ with the identity $\mathbf{I}$.

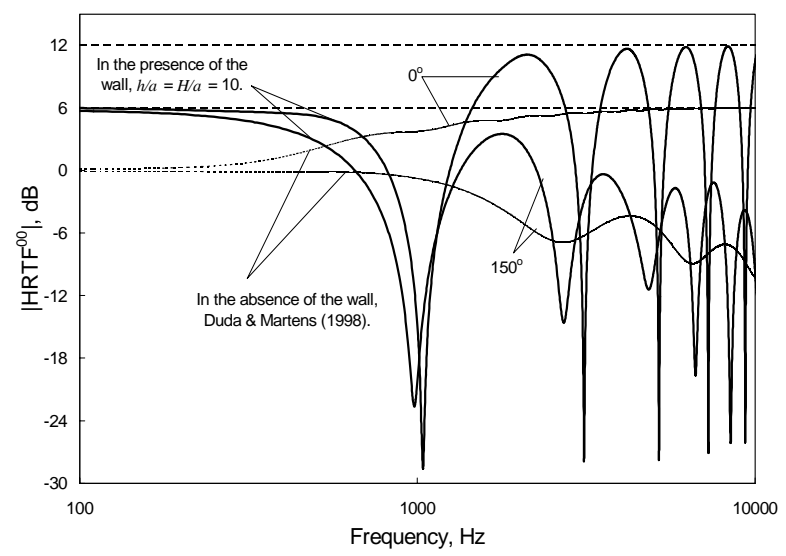

Fig. 2. $\left|\mathbb{H}^{00}\right|$ in $\mathrm{dB}$ for incidence angles $\theta_{\text {in }}=0^{\circ} \& 150^{\circ}$. The dashed line is for the theory of [2] (without a wall), while the solid line is for the present theory (with a wall). $H / a=h / a=$ $10, d / a=100, \phi=0, a=8.25 \mathrm{~cm}$.

Figs. 2 and 3 show the modulus of functions $\mathbb{H}^{00}(M)$ and $\mathbb{H}(M)$, computed for relatively far sphere locations from the

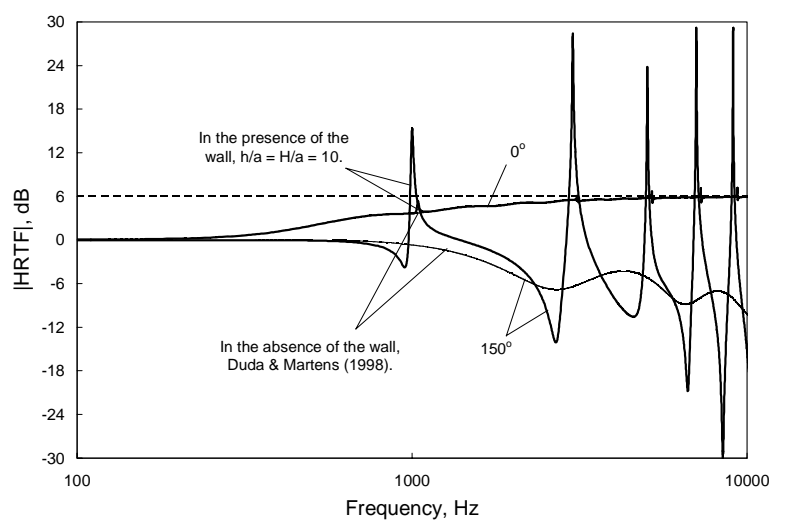

Fig. 3. $|\mathbb{H}|$ for the same conditions as in Fig. 2.

plane $(H / a=10)$ and a distant source $(d / a=100)$, for two incidence angles $\theta_{i n}=0^{\circ}$ and $150^{\circ}$, measured from the line connecting the center of the sphere and the source. For comparison, the results of [2] are also shown. Our computations show that the effect of the acoustic field scattered by the image sphere in this case does not produce any visible differences in the graphs, so the effect of wall is primarily due to the image source.

At low frequencies $|\mathbb{H}(M)| \rightarrow 1$, which corresponds to $0 \mathrm{~dB}$. This is natural for waves much larger than the head. At the same time, at low frequencies and large ranges, $\left|\mathbb{H}^{00}(M)\right| \rightarrow 2$, which corresponds to a $6 \mathrm{~dB}$ difference. This doubling is clearly due to addition of the intensities of the source and its image. From Eq. (12) we have $\mathbb{H}_{00}^{O}(O)=\left[G_{k}\left(d_{i}\right) / G_{k}(d)+1\right] \rightarrow 2$.

The frequency dependence of the HRTF is a function of the incidence angles. In addition, the influence of the wall is different on the sides of the sphere that are ipsilateral and contralateral to it. In both cases $\left|\mathbb{H}^{00}(M)\right|$ has sharp troughs. The physical explanation of these troughs is that they arise due to exact cancelation of the waves arriving with differing phase from the source and its image. To evaluate the frequencies of these peaks for large ranges we find that the difference between the distances from the real and the image source to the point under consideration is approximately $\Delta=d_{i}-d \sim 2 h^{2} / d$. The cancellation occurs when $\Delta \sim(n+1 / 2) \lambda$, where $\lambda$ is the wavelength, and $n=0,1,2, \ldots$ This gives the following expression for the cancellation frequencies, and for the trough value of the $\left|\mathbb{H}^{00}(M)\right|$ in $\mathrm{dB}$

$$
f_{n}=\frac{(2 n+1) c d}{4 h^{2}}, \quad\left|\mathbb{H}^{00}(M)\right|_{\min }=6+40 \lg \frac{h}{d} .
$$

In the case shown in Fig. $2 \& 3$ we have $c=343 \mathrm{~ms}^{-1}, d=8.25$ $\mathrm{m}, h=0.825 \mathrm{~m}$, which gives $f_{0}=1040 \mathrm{~Hz}, f_{n}=(2 n+1) f_{0}$, and $\left|\mathbb{H}^{00}(M)\right|_{\min }=-34 \mathrm{~dB}$. This agrees well with the peaks for $\theta_{i n}=0^{\circ}$. The cancellation frequencies for $\theta_{i n}=150^{\circ}$ are a bit smaller than $f_{n}$, due to the diffraction of the sound by the sphere.

The function $\mathbb{H}(M)$ for $\theta_{i n}=150^{\circ}$ substantially differs from the $\mathbb{H}(M)$ in the absence of the plane. This is because the magnitude $\left|\mathbb{H}_{00}^{M}(M, O)\right|$ has its minima shifted from those of $\left|\mathbb{H}^{00}(M)\right|$. For normal incidence, $\theta_{i n}=0^{\circ}$, the presence of the plane has little effect on $\mathbb{H}(M)$. Note also that at high frequencies the maximum increase of the pressure amplitude occurs near $\theta_{\text {in }}=0^{\circ}$, which is twice the free-field pressure. For $\left|\mathbb{H}^{00}(M)\right|$ this is $12 \mathrm{~dB}$ (see Fig. 2). 


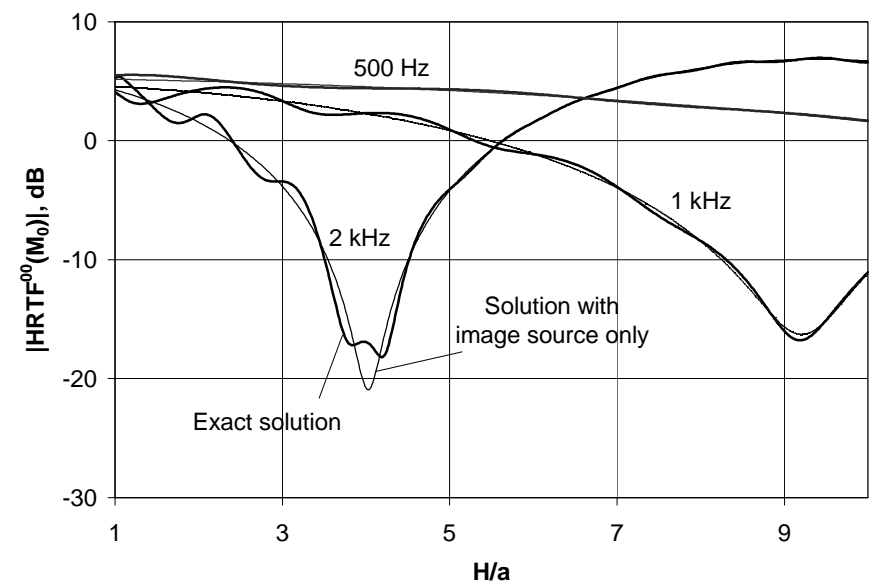

Fig. 4. Comparison of the full solution (thick) and neglecting the image sphere, but including the image source (thin). $h / a=10$, $d / a=100, \phi=0, a=8.25 \mathrm{~cm}$.

Fig. 4 shows the effect of the acoustic field scattered by the image sphere on the HRTF. Computations are performed for different frequencies and ratios $H / a$ while keeping all other parameters fixed. The value of $\left|\mathbb{H}^{00}\left(M_{0}\right)\right|$ was computed for the point on the sphere closest to the plane using both with the full $\mathbf{M}^{m}$ and with the approximation $\mathbf{M}^{m}=\mathbf{I}$. The size of $\mathbf{M}^{m}$ varies between $5 \times 5$ and $30 \times 30$. The size is chosen to obtain results that are independent of the truncation. For higher frequencies there exist oscillations of $\left|\mathbb{H}^{00}(M)\right|$ as a function of $H / a$, which are related to the ratio of the wavelength and distance between the centers of the sphere and its image. We found in all computed cases that the effect of the image sphere on the HRTF is a secondary effect compared to the effect of the image source. If the distance from the wall is equal to several times the sphere radius the effect of the image sphere may be neglected. This can be important in justifying the use of simple source imaging models for modeling room acoustics (e.g. [8])

Fig. 5 shows a computation of a case that corresponds to the "bright-spot" calculations that were shown in [2], except that there is a wall on the contralateral side of the sphere, and the source is located above the sphere along the axis of symmetry. Instead of a single bright spot, we now observe several bright bands and/or the spot, whose location and number depend upon $k a$.

\section{CONCLUSIONS}

The obtained results and preliminary study allows one to make the following conclusions about the effect of a nearby rigid boundary on the HRTF:

- The presence of a nearby rigid boundary causes a strong effect on the HRTF.

- The resulting HRTF has new peaks and troughs due to the difference in the phases of the signals coming from the source and its image.

- For distances from the wall equal to several sphere radii, an approximate approach, where one just accounts for the image source and neglects the effect of the image sphere, works very well. This may be used to justify simplified room modeling approaches such as [8].
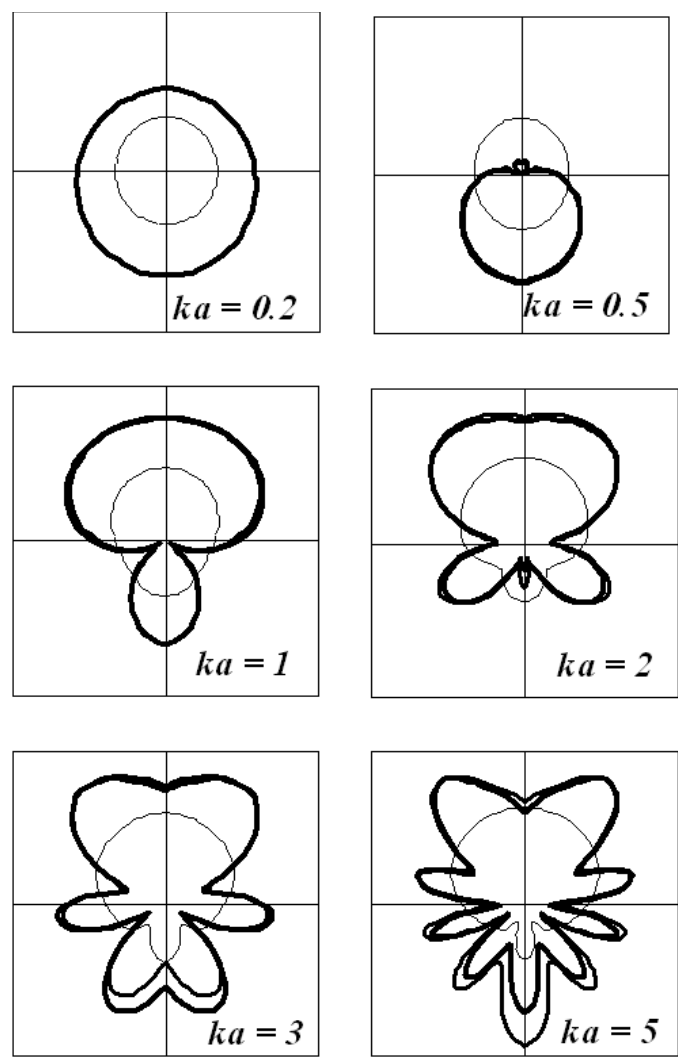

Fig. 5. Polar plots of the magnitude of $\mathbb{H}^{00}$. Thin lines correspond to the solution of [2], thicker lines to the approximate solution neglecting the image sphere, and thickest lines to the analytical solution. $H / a=2, d / a=98, h / a=100$.

- Much future work is needed to explain the psychoacoustical implications of these results.

\section{REFERENCES}

[1] C. Kyriakakis. Fundamental and Technological Limitations of Immersive Audio, IEEE Proceedings, Special Issue: Multimedia Signal Processing, Vol. 86, pp. 941-951, 1998.

[2] R.O. Duda \& W.L. Martens. Range dependence of the response of a spherical head model, J. Acoust. Soc. Am. 104, 1998, 3048-3058.

[3] A. Kulkarni, S.K. Isabelle, \& H.S. Colburn. Sensitivity of human subjects to head-related transfer-function phase spectra, J. Acoust. Soc. Am. 105, 1999 2821-2840.

[4] R.O. Duda, C. Avendano \& V.R. Algazi. An adaptable ellipsoidal head model for the interaural time difference, Proc. ICASSP 1999.

[5] P.M. Morse \& H. Feshbach. Methods of Theoretical Physics I, McGraw-Hill, 1953.

[6] M. Abramowitz \& I.A. Stegun. Handbook of Mathematical Functions, National Bureau of Standards, Wash., D.C.,1964.

[7] N.A. Gumerov \& R. Duraiswami. Multipole solutions of the Helmholtz equation, and their efficient computation, UMIACS technical report, (in preparation).

[8] J.B. Allen \& D.A. Berkeley. Image method for efficiently simulating small-room acoustics. J.Acoust.Soc.Am. 65, 1979. 